\title{
HIV-complicated meningovascular syphilis: Atypical symptoms with promising result using low molecular weight heparin
}

\author{
William C. W. Huang ${ }^{1,2 *}$, Chia-Huei Chiu ${ }^{3}$ \\ ${ }^{1}$ Department of Family Medicine, Show-Chuan Memorial Hospital, Changhua, China \\ ${ }^{2}$ Department of Life Sciences, National Chung Hsing University, Taizhong, China \\ ${ }^{3}$ Office of Medical Administration, Taichung Veterans' General Hospital, Taizhong, China \\ Email: "bill0607@gmail.com
}

Received 20 July 2012; revised 26 August 2012; accepted 20 September 2012

\begin{abstract}
Both WHO and the CDC of Taiwan have reports indicating a dramatic increase in incidence of both syphilis and HIV. With their co-infection, neurosyphilis will become a major issue, and meningovascular insults may become more complex to these Sexually Transmitted Diseases (STD). We are reporting a young individual presented with quadriparesis and initial National Institutes of Health Stroke Scale (NIHSS) greater than 20 points after having flu-like symptoms and diarrhea for a couple of days. The patient was later diagnosed as HIV-complicated meningovascular syphilis by positive blood and CSF laboratory tests. After seven-days of low molecular weight heparin (LMWH) treatment, the patient improved dramatically with NIHSS score of 2 before being transferred to HIV center for further treatment. In contrary, there is case report that demonstrates mortality after treated meningovascular syphilis with rtPA and recanalization. As the world faltten and population of HIV spreads worldwide, the prevalence of stroke in young people may increase in the upcoming century. Only scattered case reports have been presented worldwide discussing the treatment of HIV complicated with meningovascular syphilis. We are the first to rescue the stroke in the patient of HIV with syphilis by LMWH. This case report may contribute to a better treatment for infectious patient with stroke.
\end{abstract}

Keywords: Meningovascular Syphilis; HIV

\section{INTRODUCTION}

World Health Organization (WHO) estimated an annual rate of 12 million people who were infected with syphilis

"Corresponding author.
[1] worldwide with scattered outbreaks reported [2-5]. The causes of its increasing incidences include migration of people, population mixing, changes in social behaviors (such as use of internet to meet partners), the use of drugs, and reduction in safe sex practice [6]. While the issue of syphilis seems reemerged worldwide, the Center of Disease Control in Taiwan also reported HIV incidence grew 20 times more in less than a decade, from annual 800 cases in 2002 to 16,700 cases in 2008 [7].

Neurosyphilis has been a major complication of syphilic infection. Neurosyphilis can have simply headache, neck stiffness, or more moderate as photophobia, cranial nerve palsy (especially of the VII and VIII), papillaedema, psychiatric features, or seizures [8-12]. Other complicated symptoms in neurosyphilis include personality change (33\%), ataxia (28\%), and stroke (23\%) [13]. Disease may occur in the early post-primary stage or after a gap of many years.

HIV co-infection, however have not only accelerated the clinical course of neurosyphilis, increased its incidence, and complicated its presentation [14-20] such as spastic paraparesis, and medullary syndromes, etc. CDC in Taiwan showed that the incidence of HIV infection is highest at the age of 20 to 40 which accounts for $70 \%$ of infected population [7]. These young groups of co-infection with approximate $40 \%$ incidence rate of meningovascular complication [21] often onset with atypical signs and symptoms. Patients often arrive at hospital later than 3 hours of golden-period for recombinant tissue Plasminogen Ativator (rtPA) use. Or when they visit clinics early, stroke is the least impression to a physician according to its atypical signs and low risk factors. Or even worse, rtPA and recanalization may not be the optimal treatment like the old fashion stroke, and can result in an unexpected fatal vertebrobasilar occlusion [22].

As the STD co-infection spreads, the incidence and presentations of the young stroke can be altered in the new era. It thus requires further studies of the epidemio- 
logy and treatment of these STD co-infected young meningovascular disease. We hereby report the case of young HIV-complicated meningovascular syphilis with quadriparesis who respond well to Low Molecular Weight Heparin (LMWH). This non-traditional treatment highlights this important issue.

\section{THE CASE PRESENTATION}

A 40-year-old male patient was brought to the Emergency Room (ER) at 18:00 after progressive muscle weakness since morning. He had previous flu-like syndrome with abdominal pain, vomiting and diarrhea for three days. The present symptoms started with slight weakness of bilateral legs after he was awake. He felt dizzy and had difficulty in standing up from bed and walking. Those symptoms were initially thought as general malaise due to flu. At the time, the patient was alert and could communicate with family members. Later, by afternoon there were progressive neurological symptoms such as dysarthria and clumsiness of both hands. Within hours, his level of consciousness worsened progressively, and he became quadriparetic. The patient had visited local clinics once and had general gastrointestinal upset related medication due to diarrhea. Further contributory history about the patient could not be obtained from family members.

The patient had a comma scale of E2V2M3 at the time of admission. His vital signs were 145/74 mmHg, 74 bpm, 14 breaths per minute, and $36.4 \mathrm{C}$. The neurological examination showed drowsiness, severe dysarthria, dysphagia, uniform quadriparesis over all four limbs with muscle power scored three, increased tendon reflexes of all limbs (in knee, ankle, elbow and wrist), bilateral partial horizontal gaze palsy, presence of positive bilateral Babinski' s sign. The patient did not have any vertical gaze palsy, and his pupil reaction to light and accommodation were intact bilaterally. The remainder of the examination was normal. The sensory and coordination tests were not accurately evaluated. Patient's National Institutes of Health Stroke Scale (NIHSS) was greater than 20 points.

CT scan of the head without contrast was normal with mild dilated ventricles. Electrocardiographic findings showed regular sinus rhythm at the rate of 70 . The results of the complete blood count showed normal range of cell counts with slight increase in monocyte percentage $(11.6 \%)$. The results of blood chemistry showed increased creatine kinase of muscle and brain $(\mathrm{CKMB}=12)$ without elevated values of creatine phosphokinase and troponin $\mathrm{T}$ value. The level of serum sodium was marginally low (133 mmol/L), but that of potassium was within normal ranges. Arterial blood gas concentration, hepatic and renal functions were normal.
Brainstem stroke with progression was suspected and confirmed by Magnetic Resonance Imaging (MRI). It showed lesion with hyperintensity in both Fluid Attenuated Inversion Recovery (FLAIR), and Diffuse Weighted Image (DWI) at central lower and left upper pons, consistent with recent infarction (Figures 1 and 2). Since the patient has already passed the 3-hours golden period for using the recombinant tissue Plasminogen Ativator (rtPA) [23], and Low Molecular Weight Heparin (LMWH) had been also reported to significantly lower mortality rate at six months [24] wtih bleeding complication only at very high dose [25]. He was first treated with LMWH, Fraxiparin one dose subcutaneously, twice a day at intensive care unit, after informing the family about the hemorrhagic risk. Two hours later, the nuerological examination showed that the muscle power was decreased to score one. After another 6 hours, muscle power was dramtically improved to score of three and over the next 72 hours neurological signs fluctuated in severity.

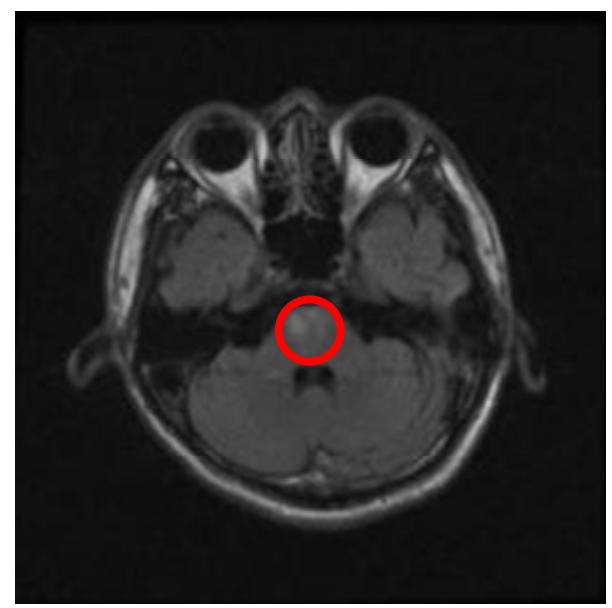

Figure 1. T2 FLAIR demonstrating the infarction at central lower pons which suggest impair vertebrobasilar artery circulation.

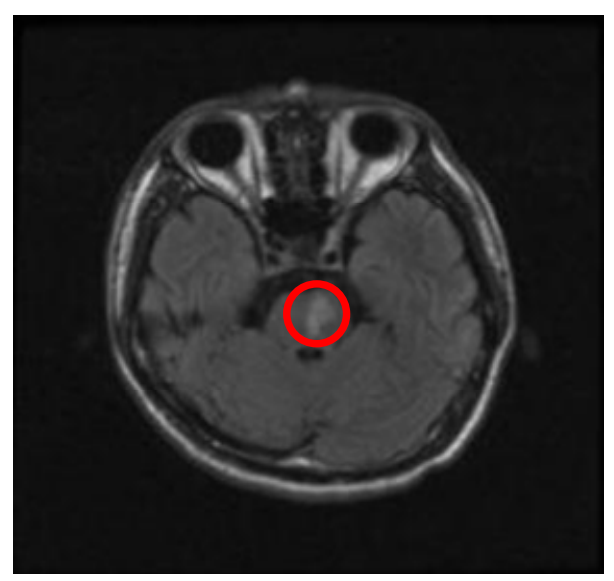

Figure 2. T2 FLAIR showing further infarction at left upper pons. 
The common risks of stroke such as coagulopathy and atherosclerosis were screened, and the data showed normal laboratory profile. Cardiac source of emboli and autoimmune disease were also ruled out.

Because monocytes showed increase in percentage, a possibility of chronic or viral infection was later considered. Tests for STD showed positive VDRL, TPHA and anti-HIV result. Central Spinal Fluid (CSF) also showed positive VDRL, and HIV-complicated meningovascular syphilitis was diagnosed. Penicilline $G$ was then given parentally according to the manual [26]. Table $\mathbf{1}$ is the summary of essential laboratory findings including both serum and CSF.

After 72 hours of LMWH treatment only (without steroid), the patient regained level of consciousness with comma scale E4V2M6 and the muscle power returned to the score of 4. Lateral gaze palsy also improved with full ranges eyeball movement. Since HIV-complicated meningovascular syphilis was impressed, history was taken from the patient again after he regained his consciousness and he recalled of having an episode of genital chancre after sextual contacts with a stranger one year ago. At that time, he received syphilis treatment and his cutaneous symptoms subsided.

Table 1. Labortory data.

\begin{tabular}{|c|c|c|c|}
\hline Serum & Item & Value & Reference \\
\hline & Anti-nuclear Ab (titer:x ) & $40 \times(-)$ & $0 \sim 40$ \\
\hline & Anti-cardiolipin IgG (GPL ) & *19.74 & $0 \sim 15$ \\
\hline & RA Factor (IU/ml ) & $<9.9$ & $0 \sim 15$ \\
\hline & S.T.S (RPR) & *1:256 × (REACTIVE) & \\
\hline & ТРНА & ${ }^{*}>1: 5120 \times$ & \\
\hline & Anti-HIV & ${ }^{*}>1: 1024 \times$ & \\
\hline & Antithrombin III & 89.2 & $80 \sim 120$ \\
\hline & Protein C & 89.1 & $70 \sim 140$ \\
\hline & Protein S & 136 & $55 \sim 160$ \\
\hline & ESR-1 hr & *51 & $0 \sim 10$ \\
\hline & C-reactive protein & 2.01 & $0 \sim 0.5$ \\
\hline & Triglyceride & 58 & $<150$ \\
\hline & Cholesterol & 174 & $<200$ \\
\hline & LDL-C & 128 & $0 \sim 150$ \\
\hline \multirow[t]{18}{*}{ Note: ${ }^{*}$ Indicates abnormality in value. CSF } & Appearance & +/- TURBID & \\
\hline & Color & PALE & \\
\hline & India stain & NOT FOUND & \\
\hline & Lymphocyte $^{\circ}(\%)$ & 98 & \\
\hline & Polymorphonuclear cell (\%) & 2 & \\
\hline & Protein(Pandy’s) & ${ }^{*} 1+$ & $(-)$ \\
\hline & $\mathrm{RBC}(/ \mathrm{ul})$ & $7-9$ & \\
\hline & WBC count (/ul) & *27 & $0 \sim 5$ \\
\hline & Protein (mg/dl) & ${ }^{*} 122$ & $15 \sim 45$ \\
\hline & GLU (mg/dl) & ${ }^{*} 42$ & $40 \sim 75$ \\
\hline & Albumin (\%) & 56.4 & \\
\hline & Alpha-1 (\%) & 11.7 & \\
\hline & Alpha-2 (\%) & 8.0 & \\
\hline & Beta (\%) & 14.4 & \\
\hline & Cryptococcal Ag & NEGATIVE & \\
\hline & Gamma (\%) & 9.6 & \\
\hline & M-peak & $(-)$ & \\
\hline & VDRL & *1:8 × (POSITIVE) & \\
\hline
\end{tabular}


After receiving one-week LMWH, the patient was stable in the level of consciousness and was oriented to person, time and place. Muscle power was fully recovered without previous hyperreflexia. NIHSS was scored two with only persisting mild dysarthira and dysphagia. After being hospitalized for 14 days, he was transferred to Taiwan University AIDS Center for controlled management of HIV.

\section{DISCUSSION}

Meningovascular syphilis, a clinical presentation of tertiary syphilis, is caused by an obliterative endarteritis, involving the vaso vasorum of the central nervous system. The pathologic finding is diffuse lymphocytic infiltration in the perivascualr space and thickening of the vascular wall. On the other hand, the pathological change of vascular wall in hypertensive atherosclerosis has been reported with fibroblastic and collagenous proliferation of the intima. Based on the pathological difference, it may explain the findings with or without rapid or fluctuated progression due to ongoing inflammatory changes in comparison to atherosclerosis. In addition, the process of meningovascular syphilis frequently occurrs in medium size vessels (Heubner's arteritis) and small intracranial vessels (Nissl-Alzheimer arteritis) which may lead to cerebrovascular thrombosis and ischemic infarction due to narrowing of lumen [21], and involving predominantly in middle cerebral artery and then in basilar artery [19]. Our case also had ischemic infarction involving basilar artery.

HIV co-infection makes the meningovascular syphilis more complex. In many previous case reports of simple meningovascular syphilis, prodromal clinical course runs several weeks to months before a focal neurological deficit can be described [21,27-29]. The patient in this report with co-infection did not have persistently prodromal symptoms prior to onset of quadriparesis, although he had reported several flu-like episodes over the past few months. Also, the onset of meningovascular insult in our case was less than a year, which was much shorter in duration as compared to the classical neurosyphilis infection. His condition was also complicated by acute onset of abdominal pain with vomiting and diarrhea for three days. Dehydration and underlying persisted endarteritis may cause such ischemic stroke.

The clinical and pathological findings of the meningovascular insults in patient with combined STD are often atypical and delayed diagnosis. The treatment does deserve further attention, especially the younger population between 20 and 40, who are prone to STD. As younger age of population with co-infection of different STD had less chronic illness may retain a reversible function of vessel wall accommodation and respond better to treatment. Risk of hemorrhage may be greatly reduced under the short term use of anticoagulant in the specific group.

Given that younger patient with co-infected STDs commonly delays the recognition of meningovascular insults and miss the early chance to use rtPA, short term use of LMWH may be an alternative treatment choice. Flint $e t$ al. also reportted the case of a male at his $40 \mathrm{~s}$ presented with left hemiparesis and dysarthria, who was later proved to have menigovascualr syphilis with brainstem infarct. This HIV-negative patient received intravenous heparin therapy. In two-month follow up, he was not hemiparetic [30]. On the other hand, Feng et al. reported a young patient with meningovascular syphilis, who was treated with rtPA and resulted in a fatal vertebrobasilar occlusion [22].

As the population of co-infected STDs increases, the clinicians should be aware of the possibility of having STD associated meningovascular infarct among young population. These groups of patients are usually presented with atypical symptoms and fluctuated signs, and often miss the chance of rtPA treatment. Thus, further studies of its epidemiology and more effective treatment are warranted. Our case presentation may provide an alternative treatment for the patient with co-infected STDs.

\section{REFERENCES}

[1] World Health Organization (WHO). http://www.who.int./

[2] Borisenko, K.K., Tichonova, L.I. and Renton, A.M. (1999) Syphilis and other sexually transmitted infections in the Russian Federation. International Journal of STD \& AIDS, 10, 665-668. doi:10.1258/0956462991913240

[3] Van den Hoek, A., Yuliang, F., Dukers, N.H., et al. (2001) High prevalence of syphilis and other sexually transmitted diseases among sex workers in China: Potential for fast spread of HIV. AIDS, 13, 753-759. doi:10.1097/00002030-200104130-00011

[4] Sugihantono, A., Slidell, M., Syaifudin, A., et al. (2003) Syphilis and HIV prevalence among commercial sex workers in central java, Indonesia: Risk-taking behavior and attitudes that may potentiate a wider epidemic. AIDS Patient Care STDS, 17, 595-600. doi:10.1089/108729103322555980

[5] Ashton, M., Sopwith, W., Clark, P., McKelvey, D., Lighton, L. and Mandal, D. (2003) An outbreak no longer: Factors contributing to the return of syphilis in Greater Manchester. Sexually Transmitted Infections, 79, 291-293. doi:10.1136/sti.79.4.291

[6] Ciesielski, C.A. (2003) Sexually transmitted diseases in men who have sex with men: An epidemiologic review. Current Infectious Disease Reports, 5, 145-152. doi:10.1007/s11908-003-0051-5

[7] Center for Disease Control (CDC). www.cdc.gov.tw

[8] Cintron, R. and Pachner, A.R. (1994) Spirochetal diseases of the nervous system. Current Opinion in Neuro- 
logy, 7, 217-222. doi:10.1097/00019052-199406000-00007

[9] Dalal, P.M. and Dalal, K.P. (1992) Cerebrovascular manifestations of neurosyphilis. Journal of the Association of Physicians of India, 40, 603-606.

[10] Hutto, B. (2001) Syphilis in clinical psychiatry: A review. Psychosomatics, 42, 453-460. doi:10.1176/appi.psy.42.6.453

[11] Katz, D.A., Berger, J.R. and Duncan, R.C. (1993) Neurosyphilis: A comparative study of the effects of infection with human immunodeficiency virus. Archives of Neurology, 50, 243-249. doi:10.1001/archneur.1993.00540030009006

[12] Primavera, A., Solaro, C., Cocito, L., et al. (1998) Status epilepticus as the presenting sign of neurosyphilis. Epilepsia, 39, 1367-1369. doi:10.1111/j.1528-1157.1998.tb01339.x

[13] Cintron, R. and Pachner, A.R. (1994) Spirochetal diseases of the nervous system. Current Opinion in Neurology, 7, 217-222. doi:10.1097/00019052-199406000-00007

[14] Funnye, A.S. and Akhtar, A.J. (2003) Syphilis and human immunodeficiency virus co-infection. Journal of the $\mathrm{Na}$ tional Medical Association, 95, 363-382.

[15] Flores, J.L. (1995) Syphilis: A tale of twisted treponemes. The Western Journal of Medicine, 163, 552-559.

[16] Strom, T. and Schneck, S.A. (1991) Syphilitic meningomyelitis. Neurology, 41, 325-326. doi:10.1212/WNL.41.2_Part_1.325

[17] Tyler, K.L., Sandberg, E. and Baum, K.F. (1994) Medical medullary syndrome and meningovascular syphilis: A case report in an HIV-infected man and a review of the literature. Neurology, 44, 2231-2235. doi:10.1212/WNL.44.12.2231

[18] Katz, D.A., Berger, J.R. and Duncan, R.C. (1993) Neurosyphilis: A comparative study of the effects of infection with human immunodeficiency virus. Archives of Neurology, 50, 243-249. doi:10.1001/archneur.1993.00540030009006

[19] Musher, D.M., Hamill, R.J. and Baughn, R.E. (1990) Effect of human immunodeficiency virus (HIV) infection on the course of syphilis and on the response to treatment. Annals of Internal Medicine, 113, 872-881.

[20] Simpson, D.M. and Tagliati, M. (1994) Neurologic mani- festations of HIV infection. Annals of Internal Medicine, 121, 769-785.

[21] Pezzini, A., Gulletta, M., Pinelli, L., et al. (2001) Meningovascular Syphilis-A vascular syndrome with typical features. Cerebrovascular Diseases, 11, 352-353. doi:10.1159/000047667

[22] Feng, W.W., Caplan, M., Matheus, M.G., et al. (2009) Meningovascular syphilis with fatal vertebrobasilar occlusion. The American Journal of the Medical Sciences, 338, 169-171.

[23] Adams, H.P., Brott, T.G., Crowell, R.M., et al. (1994) Guidelines for the management of patients with acute ischemic stroke: A statement for healthcare professionals from a special writing group of the Stroke Council. American Heart Association, 90, 1588-1601. doi:10.1161/01.CIR.90.3.1588

[24] Kay, R., Wong, K.S., Yu, Y.L., et al. (1995) Low-molecular-weight heparin for the treatment of acute ischemic stroke, The New England Journal of Medicine, 333, 15881593. doi:10.1056/NEJM199512143332402

[25] Diener, H.C., Ringelstein, E.B., von Kummer, R., et al. (2001) Treatment of acute ischemic stroke with the low-molecular-weight heparin certoparin: Results of the TOPAS trial: Therapy of patients with acute stroke (TOPAS) investigators. Stroke, 32, 22-29. doi:10.1161/01.STR.32.1.22

[26] Adams and Victor's Manual of Neurology, 7th Edition. Ch. 32 Infections of The Nervous System and Sarcoidosis.

[27] Standaert, D., Galetta, S. and Atlas, S. (1991) Meningovascular syphilis with a gumma of the midbrain, Journal of Neuro-Ophthalmology, 113, 139-143.

[28] Johns, D.R., Tierney, M. and Parker, S.W. (1987) Pure motor hemiplesia due to meningovascular neurosyphilis. Archives of Neurology, 44, 1062-1065. doi:10.1001/archneur.1987.00520220060018

[29] Holmes, M.D., Brant-Zawadzki, M.M. and Simon, R.P. (1984) Clinical features of meningovascular syphilis. Neurology (Cleveland), 34, 553-556. doi:10.1212/WNL.34.4.553

[30] Flint, A.C., Liberato, B.B., Anziska, Y., et al. (2005) Meningovascular syphilis as a cause of basilar artery stenosis. Neurology, 64, 391-392. doi:10.1212/01.WNL.0000149758.57386.B8 